and B cell activation. Iscalimab showed clinical efficacy in a Proof of Concept randomised controlled trial at a dose of $10 \mathrm{mg} / \mathrm{kg}$ intravenously (IV), whereas subcutaneous (SC) dosing at $3 \mathrm{mg} / \mathrm{kg}$ was associated with unexpectedly low plasma concentrations and reduced efficacy, likely due to efficient pre-systemic target-mediated clearance.

Objectives: To test IV versus SC loading doses of iscalimab followed by SC maintenance dosing, as a means of achieving target drug exposure and clinical efficacy.

Methods: Patients with clinically active pSS [EULAR Sjögren's Syndrome Disease Activity Index (ESSDAI) $\geq 6$ ] were randomised to receive either $600 \mathrm{mg}$ SC iscalimab weekly on 4 occasions, followed by $300 \mathrm{mg}$ SC weekly until week 12 , or a single IV dose of $10 \mathrm{mg} / \mathrm{kg}$ iscalimab on study Day 1, followed by $300 \mathrm{mg}$ SC weekly until week 12 . Subjects and investigator staff remained blinded to study treatment allocation until first dosing.

Results: Twenty-five patients were randomised; 13 in the SC loading and 12 in the IV loading arms. Baseline characteristics were similar to the previous phase Ila cohorts with mean ESSDAI scores of 12.7 (SD 6.1) and 10.4 (5.9) in the SC and IV loading arms respectively. In Arm 1 (SC) and Arm 2 (IV), the mean trough plasma concentrations were 169 $\mu \mathrm{g} / \mathrm{mL}$ (SD 64.1, CV 38\%) and $135 \mu \mathrm{g} / \mathrm{mL}$ (SD 70.9, CV 53\%) on Day 85 , respectively. Both values were well above levels previously reported to be sufficient for suppression of germinal centre development and $T$ dependent antigen responses in cynomolgus monkeys. Consistent with this finding, clinically important improvements were seen in both arms with a mean decrease in ESSDAl scores of -5.5 (+/- SD: 5.5$)$ and -7.6 (+/- 7.1) points from baseline to Day 85 in the SC and IV dosing arms. Improvements were also seen in EULAR Sjögren's Syndrome Patient Reported Index (ESSPRI) scores: -1.67 (+/- 1.8) and -1.17 (+/- 2.3), respectively. Other secondary efficacy outcomes showed similar patterns of improvement. Treatment with iscalimab was associated with a reduction in the germinal centre-related serum biomarker CXCL13 in both groups. Overall, iscalimab was safe and well-tolerated with no new safety signal emerging. One subject experienced three SAEs (hemarthrosis, worsening of right knee pain and swelling requiring arthroscopy) in the safety followup period, all unrelated to study drug.

Conclusion: These results further support the safety and efficacy of iscalimab in pSS and the suitability of SC dosing for future development.

Disclosure of Interests: Benjamin Fisher Consultant for: Novartis, Roche, Medlmmune, Bristol-Myers Squibb, Antónia Szántó: None declared, Wan Fai $\mathrm{Ng}$ : None declared, Michele Bombardieri Grant/research support from: Celgene, Consultant for: Medimmune, Maximilian Posch: None declared, Athena Papas Grant/research support from: Novartis, Consultant for: Novartis, Arwa Farag: None declared, Thomas Daikeler: None declared, Bettina Bannert: None declared, Alan Kivitz Shareholder of: Novartis, Consultant for: Abbvie, Janssen, Pfizer, UCB, Genzyme, Sanofi, Regeneron, Boehringer Ingelheim, Sun Pharma Advanced Research, Flexion., Paid instructor for: Celgene, Horizon, Merck, Novartis, Pfizer, Genzyme, Sanofi, Regeneron, Speakers bureau: Celgene, Horizon, Merck and Genetech, Flexion, Steven Carsons Grant/research support from: Novartis, David Isenberg: None declared, Francesca Barone Grant/research support from: GlaxoSmithKline, Roche, UCB Pharma, Actelion, ONO Pharmaceutical, Consultant for: GlaxoSmithKline, Roche, Actelion, ONO Pharmaceutical, Simon J. Bowman Grant/research support from: Previously

UCB Pharma (to University of Birmingham) and Roche, Consultant for: 2016-7: Novartis, Mitsubishi Tanabe Pharma

2017-8: AstraZeneca, Medlmmune, GFK, Xtlbio, ONO Pharmaceutical 2018-9: Novartis, AstraZeneca, UCB Pharma, Pascal Espie Employee of: Novartis, Grazyna Wieczorek Employee of: Novartis, Pierre Moulin Employee of: Novartis, David Floch Employee of: Novartis, Cyrielle Dupuy Employee of: Novartis, Amanda Nguyen Employee of: Novartis, Andrew Wright Shareholder of: Novartis, Employee of: Novartis, Michael Rotte Employee of: Novartis, James Rush Employee of: Novartis, Peter Gergely Employee of: Novartis

DOI: 10.1136/annrheumdis-2019-eular.4277

\section{FRI0175 \\ PREDICTORS OF LOW DISEASE ACTIVITY AND CLINICAL REMISSION FOLLOWING BELIMUMAB TREATMENT IN SYSTEMIC LUPUS ERYTHEMATOSUS}

loannis Parodis ${ }^{1,2}$, Petter Johansson ${ }^{1,2}$, Alvaro Gomez $^{1,2}$, Sofia Soukka ${ }^{1,2}$ Sharzad Emamikia ${ }^{1,2}$, Katerina Chatzidionysiou ${ }^{1,2} .{ }^{1}$ Karolinska Institutet, Division of Rheumatology, Department of Medicine, Stockholm, Sweden; ${ }^{2}$ Karolinska University Hospital, Rheumatology, Stockholm, Sweden

Background: Post-hoc analyses of the pivotal phase III clinical trials of belimumab BLISS-52 and BLISS-76 have revealed superiority of belimumab over placebo in systemic lupus erythematsus (SLE) patients with high baseline disease activity, positive anti-double stranded (ds)DNA titres and low complement levels, as well as in patients receiving corticosteroids (1). Later, real-life observations demonstrated that established organ damage prior to treatment initiation predicted reduced belimumab efficacy based on the SLE Responder Index 4 (SRI-4) (2), which was recently corroborated in a post-hoc analysis of data from the BLISS trials (3). From a clinical point of view, clinical remission and low disease activity are more meaningful targets than reduced SLE activity (SRI-4).

Objectives: To identify predictors of low disease activity and clinical remission following belimumab treatment in patients with SLE.

Methods: SLE patients who received belimumab $10 \mathrm{mg} / \mathrm{kg}(\mathrm{N}=563)$ in the BLISS-52 and BLISS-76 clinical trials were surveyed. Access to data was granted by GlaxoSmithKline. The performance of baseline factors in predicting attainment of low disease activity defined as Lupus Low Disease Activity State (LLDAS) (4) or clinical remission defined as clinical (c)SLE$\mathrm{DAI}-2 \mathrm{~K}=0$ at week 52 from treatment initiation was evaluated using logistic regression. Organ damage was assessed using the SLICC/ACR Damage Index (SDI).

Results: We demonstrated a negative impact of established organ damage on attainment of LLDAS (SDI>0; OR: 0.44 ; $95 \% \mathrm{Cl}$ : $0.22-0.90$; $\mathrm{P}=0.024$ ) and the primary LLDAS condition, i.e. SLEDAI- $2 \mathrm{~K} \leq 4$ with no renal activity, pleurisy, pericarditis or fever (SDI>1; OR: $0.46 ; 95 \% \mathrm{Cl}$ : 0.27-0.77; $\mathrm{P}=0.004)$; cognitive impairment/psychosis was found to mainly account for the latter association. Baseline SDI scores $>1$ predicted failure to attain cSLEDAI-2K=0 (OR: $0.53 ; 95 \% \mathrm{Cl}$ : $0.30-0.94 ; \mathrm{P}=0.030$ ), with cutaneous damage mainly driving this association. Anti-dsDNA positivity increased (OR: 1.82; 95\% Cl: 1.08-3.06; $\mathrm{P}=0.025$ ) and cardiovascular damage reduced (OR: $0.13 ; 95 \% \mathrm{Cl}: 0.02-0.97 ; \mathrm{P}=0.047$ ) the probability to attain CSLEDAI-2K=0 with the daily prednisone equivalent intake restricted to $\leq 7.5 \mathrm{mg}$

Conclusion: Belimumab might be expected to be more efficacious in inducing low disease activity and clinical remission in SLE patients with limited or no organ damage accrued prior to treatment initiation. Patients with positive anti-dsDNA titers might be more likely to achieve clinical remission along with limited or no corticosteroid use. The findings contribute to a better selection of SLE patients expected to benefit from belimumab, and provide useful information towards refinement of SLE treatment recommendations.

\section{REFERENCES:}

[1] van Vollenhoven RF, Petri MA, Cervera R, et al. Ann Rheum Dis 2012

[2] Parodis I, Sjowall C, Jonsen A, et al. Autoimmun Rev 2017.

[3] Parodis I, Gomez A, Emamikia S, et al. Ann Rheum Dis 2019.

[4] Franklyn K, Lau CS, Navarra SV, et al. Ann Rheum Dis 2016.

Acknowledgement: The authors would like to thank GlaxoSmithKline (Uxbridge, UK) for granting access to the data from the BLISS-52 and BLISS-76 trials (ClinicalTrials.gov identifiers NCT00424476 and NCT00410384, respectively) through the Clinical Study Data Request (CSDR) consortium.

Disclosure of Interests: None declared

DOI: 10.1136/annrheumdis-2019-eular.6376

\section{FRI0176 PHASE 2, DOUBLE-BLIND, RANDOMIZED, PLACEBO- CONTROLLED STUDY OF A REVERSIBLE B CELL INHIBITOR, XMAB ${ }^{\circledR} 5871$, IN SYSTEMIC LUPUS ERYTHEMATOSUS (SLE)}

Joan Merrill $^{1}$, Joshua June ${ }^{2}$, Fotios Koumpouras ${ }^{3}$, Wambui Machua ${ }^{4}$, Mohammad Faisal Khan $^{5}$, Anca Askanase ${ }^{6}$, Arezou Khosroshahi ${ }^{7}$, Saira Sheikh ${ }^{8}$, Judith A. James ${ }^{1}$, Joel Guthridge ${ }^{1}$, Gaurav Rathi ${ }^{9}$, Bart Burington ${ }^{9}$, Paul Foster ${ }^{9}$, Debra Zack ${ }^{10} .{ }^{1}$ Oklahoma Medical Research Foundation, Oklahoma City, United States of America; ${ }^{2}$ Great Lakes Center of Rheumatology, Lansing, United States of America; ${ }^{3}$ Yale University, New Haven, United States of America; ${ }^{4}$ Piedmont Physicians Atlanta Rheumatology, Atlanta, United States of America; ${ }^{5}$ Arthritis and Rheumatology Center of Oklahoma, PLLC, Oklahoma City, United States of America; ${ }^{6}$ Columbia University Medical Center, New York. United States of America; ${ }^{7}$ Emory University, Atlanta, United States of America; ${ }^{8}$ University of North Carolina at Chapel Hill, Chapel Hill, United States of America; ${ }^{9}$ Xencor Inc., San Diego, United States of America; ${ }^{10}$ Xencor Inc, Clinical Development, San Diego, United States of America

Background: $\mathrm{XmAb5871}$ is a humanized anti-CD19 antibody Fc-engi-

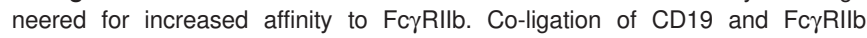
inhibits B lineage cells key to SLE pathogenesis. 